\title{
Fast Reduction of Potential Fields Measured Over an Uneven Surface to a Plane Surface
}

\author{
P. S. Naidu and M. P. Mathew
}

\begin{abstract}
The present work is aimed at rapid reduction of the gravity and magnetic fields observed over an uneven surface to a horizontal plane. The approach suggested here is to estimate the Fourier transform of the potential field over an imaginary horizontal plane lying entirely above the ground surface and impose boundary conditions; namely, the solution must satisfy the observed field over the ground surface and vanish over an infinite hemisphere. The desired Fourier transform is obtained in an iterating manner. A 2-D FFT algorithm can considerably reduce the computational burden. The FFT approach cannot be used unless the discrete data is available on a rectangular grid. If the observations are scattered, interpolation to the nearest grid point will have to be carried out. Interpolation introduces marginal increase in the rms error. The iterating approach is about 10 times faster than the least squares approach.
\end{abstract}

\section{INTRODUCTION}

$I_{n}^{N}$ $\mathrm{N}$ geophysical surveys, and in particular, in gravity and magnetic surveys, the observation stations are often controlled by topography, accessibility, and the distance to the base station to which one has to return for frequent calibration. These factors naturally control the distribution of the observation stations; mostly along the existing motorable tracks. In aerial low-altitude mineral surveys the aircraft is often flown at a constant height (e.g., $50 \mathrm{~m}$ ) above the topographic surface. Consequently, the flight lines, specially in hilly terrain, are undulating in the vertical plane, though practically parallel in the horizontal plane. Thus, the observation stations may be considered as unevenly distributed on an irregular topographic surface or on flight lines lying on an irregular surface parallel to the topographic surface below. Such observations of the gravity and magnetic field are inadequate for modern quantitative methods that require discrete observations over a square grid on a horizontal plane. The earliest work on the subject of the continuation of the potential field measured on a curved line to a horizontal line above the curve line was by Strakhov and Devitsyn [1], who used the method of successive approximations for the solution of an integral equation of first order. Their method works well when the surface undulations are weak. Tsirul'skiy [2] reduced the problem to solving of an integral equation

Manuscript received November 31, 1993

P. S. Naidu is with the Department of Electrical Communication Engineering, Indian Institute of Science, Bangalore 560012, India.

M. P. Mathew is with Airborne Mineral Survey and Exploration, Geological Survey of India, Bangalore 560001, India.

IEEE Log Number 9400439. of the second kind in the frequency domain. An algorithm to solve the integral equation by a method of successive approximation is given in [3]. Bhattacharya and Chen [4] have formulated the problem in terms of the Fredholm integral equation and gave a series solution in the space domain [5]. Hansen and Miyazaki [6] modified the method given in [4] for better accuracy. Henderson and Cordell [7] have used finite harmonic series expansion of the observed data and then, using the estimated coefficients, they were able to reduce the field to a plane surface. The French workers used the Backus and Gilbert inverse problem formalism for the continuation of the potential field from an uneven surface to a plane surface [8], [9]. Parker and Klitford [10] have used the Schwartz-Christoffel transformation to map an uneven track of a bottom-towed magnetometer to a straight line.

The present paper addresses itself to the problem of reducing the field observed over an irregular surface onto a horizontal plane above the surface of observation. The approach adopted here is similar to that in [1] and [2], but it is valid both for two- and three-dimensional problems and it uses a fast computational algorithm. The computations require the repeated application of a 2-D fast Fourier transform, thus making the task of reduction very fast compared to the space-domain methods described in [4]$[10]$ and currently used in industry. However, the major limitation of the present approach lies in the requirement that the $x$ and $y$ coordinates of a measurement station must lie on a square grid, though the $z$ coordinate may take any arbitrary value (see Fig. 1). Only then can one exploit the high speed of the FFT algorithm. From a practical point of view this does not pose a serious problem as it is a common practice to generate gridded data from the scattered observations. Using such an approach we have obtained accurate reduction even when the observation stations were scattered, but a sufficiently large number of them were available. The present paper is divided into five sections, In the next section we briefly review the least-squares inversion method and study its performance when the available data is randomly scattered. In Section III we describe the theory of an iterating FFT-based method and in Section IV we provide a few illustrations as well as a synthetic example. In Section $V$ we provide a practical example. We shall also compare the accuracy and speed of the present method with that of the leastsquares method. 


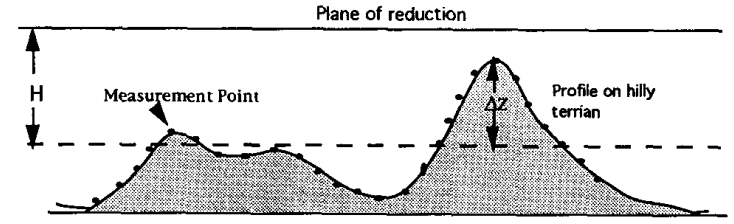

Fig. 1. Profile of observation stations over a hilly terrain. The observed field is now reduced to a horizontal plane just above the highest point in the area of survey.

\section{LEAST-SQuARES INVERSION}

We shall assume that all observation points are scattered in a three-dimensional space but that they all lie on a smooth plane representing a topographic surface. The data points when projected onto a horizontal plane are scattered over the plane. Let $f(x, y, H)$ be the potential field on a horizontal plane $H$ units above the mean observation surface and $F(u, v, H)$ be its 2-D Fourier transform (see Fig. 1). We shall continue the unknown field on the horizontal plane downward to every point of observation,

$$
\begin{aligned}
f\left(x_{i}, y_{i}, H+\Delta z_{i}\right) & \\
= & \frac{1}{4 \pi^{2}} \int_{-\infty}^{\infty} \int_{-\infty}^{\infty} F(u, v, H) e^{-s\left(H+\Delta z_{i}\right)} \\
& \cdot e^{j\left(u x_{i}+v y_{i}\right)} d u d v, \quad i=0,1, \cdots N-1 .
\end{aligned}
$$

In above equation the unknown quantity is $F(u, v, H)$, which may be discretized over a finite domain with the assumption that it vanishes outside this domain. For the sake of simplicity we shall assume the domain is rectangular, $2 a \times 2 b$, and it is divided into $(2 p+1) \times(2 q+$ 1) cells. We shall discretize the integral in (1) and write

$$
\begin{aligned}
f\left(x_{i}, y_{i}, H-\Delta z_{i}\right) & =\frac{1}{P Q} \sum_{k=-p}^{p} \sum_{l=-q}^{q} F\left(\frac{2 \pi}{P} k, \frac{2 \pi}{Q} l, H\right) \\
& \cdot \exp \left[-\left(H-\Delta z_{i}\right) \sqrt{\left(\frac{2 \pi}{P} k\right)^{2}+\left(\frac{2 \pi}{Q} l\right)^{2}}\right] \\
& \cdot \exp \left[j\left(\frac{2 \pi}{P} k x_{i}+\frac{2 \pi}{Q} l y_{i}\right)\right]
\end{aligned}
$$

where $P=2 p+1$ and $Q=2 q+1$. Define following matrices:

$$
\begin{aligned}
& \mathbf{f}= \operatorname{col}\left\{f\left(x_{i}, y_{i}, H-\Delta z_{i}\right), \quad i=1,2,3 \cdots N\right\} \\
& \text { Size: } N \times 1 \\
& \mathbf{A}= \frac{1}{P Q}\left\{\exp \left[-\left(H-\Delta z_{i}\right) \sqrt{\left(\frac{2 \pi}{P} k\right)^{2}+\left(\frac{2 \pi}{Q} l\right)^{2}}\right]\right. \\
& \cdot \exp \left[j\left(\frac{2 \pi}{P} k x_{i} \frac{2 \pi}{Q} l y_{i}\right)\right] \quad i=1,2 \cdots N \\
& k=-p,-p+1 \cdots p-1, p \\
&\quad l=-q,-q+1 \cdots q-1, q\} \\
& \text { Size: } N \times(2 p+1)(2 q+1)
\end{aligned}
$$

Table I

\begin{tabular}{|l|l|l|l|l|l|l|l|}
\hline $\begin{array}{l}\text { Number of } \\
\text { observations. }\end{array}$ & 2500 & 2000 & 1500 & 1000 & 750 & 600 & 450 \\
\hline rms error (\%) & 1.29 & 1.49 & 1.78 & 1.81 & 3.20 & 6.10 & 127 \\
\hline $\begin{array}{l}\text { CPU Time (min) } \\
\text { on Vax 750. }\end{array}$ & 125 & 105 & 89 & 62 & 50 & 42 & 33 \\
\hline
\end{tabular}

and

$$
\begin{aligned}
\mathbf{F}=\operatorname{col} & \left\{F\left(\frac{2 \pi}{P} k, \frac{2 \pi}{Q} l, H\right),\right. \\
& k=-p,-p+1 \cdots p-1, p \\
l & =-q,-q+1 \cdots q-1, q\} .
\end{aligned}
$$

Size: $(2 p+1)(2 q+1)$

Equation (2) in matrix version becomes $\mathbf{f}=\mathbf{A F}$. We should be able to solve the above system of linear equations for the unknowns contained in vector $F$. Naturally, we must have $N \geq(2 p+1)(2 q+1)$; in the presence of noise a reliable solution is obtained only when $N \gg(2 p$ $+1)(2 q+1)$. A least-squares solution of $(2)$ is given by

$$
\mathbf{F}=\left(\mathbf{A}^{H} \mathbf{A}\right)^{-1} \mathbf{A}^{H} \mathbf{f}
$$

where the superscript $H$ stands for the hermitian transpose. We have evaluated (3) on a Vax 750. The computation time required for different data sizes is shown in Table I.

\section{A. Numerical Example}

A ground magnetic survey over an area of $140 \times 140$ units (arbitrary units) over an undulating terrain was simulated. The field at randomly scattered stations uniformly covering the entire area was calculated. The magnetic field is caused by four semiinfinite prisms. A map of the undulating surface including the stations and an outline of magnetic targets are shown in Fig. 2. The undulating surface was generated mathematically using the equation

$$
\begin{aligned}
\Delta z(x, y)= & 4.0+2.0 \cos \left[\frac{x^{2}}{\sqrt{x^{2}+y^{2}+16}}\right] \\
& +\sin \frac{\sqrt{x^{2}+y^{2}+16}}{y^{2}+4} .
\end{aligned}
$$

The height of the observation stations varied from 1.1 to 7.0 units. First, the scattered field measurements were used for reduction. The least-squares inversion approach was used to reconstruct the field at a height of 7.5 units, just above the highest point. The mean-square error between the reconstructed field and the actual (computed) field is tabulated in Table I.

The error is expressed in percentage relative to the peakto-trough anomaly amplitude of 65.5 gamma. The rms error is found to increase rapidly when the number of stations is reduced below 600 . 


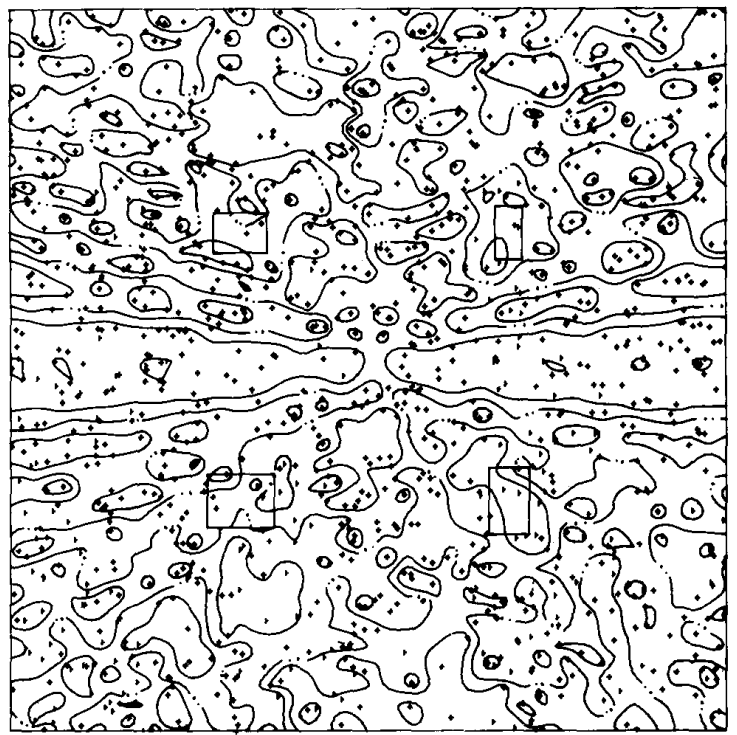

Fig. 2. A map of the undulating surface including the observation stations $(+)$ and an outline of magnetic targets.

\section{FFT-Based Method}

Let $f_{0}(x, y, \Delta z)$ be the potential field (gravity or magnetic) observed over a known but arbitrary surface $\Delta z(x$, $y$ ). We shall assume that the $x$ and $y$ coordinates of each observation station lie on a regular grid; however, the $z$ coordinate is arbitrary. Where this is not true we shall assume that through interpolation, gridded digital magnetic data has been prepared. The data points, when projected onto a horizontal plane, fall on a regular grid of points. Only the height of a data point is irregular. Let $f(x, y, h)$ be the desired field on a horizontal plane $H$ units above the mean observation surface $[H>\Delta z(x, y)]$. Now we shall continue the field downward to the observation surface and require that the downward continued field must satisfy the observed field

$$
\begin{aligned}
f_{0}(x, y, \Delta z)= & \frac{1}{4 \pi^{2}} \int_{-}^{+} \int_{\pi}^{\pi} F(u, v, h) e^{s h(1-\Delta z(x, y) / h)} \\
& \cdot e^{j(u x+v y)} d u d v
\end{aligned}
$$

where $F(u, v, h)$ is the Fourier transform of the desired field $f(x, y, h)$ and $s=\sqrt{u^{2}+v^{2}}$. Note that the representation of the potential field given in (4) satisfies the Laplace equation and the vanishing boundary condition at $+\infty$. Next, we express $\Delta z(x, y) / h=\epsilon \eta(x, y)$ where $\epsilon<$ 1 and $\eta(x, y)$ is a normalized surface, that is,

$$
\frac{1}{4 X Y} \sum_{-X}^{X} \sum_{-Y}^{Y} \eta^{2}(x, y)=1, \quad X, Y \rightarrow \infty
$$

Use the following expansions:

$$
\begin{aligned}
e^{-s \Delta z(x, y)} & =\sum_{k=0}^{\infty} \frac{(-1)^{k} s^{k} \Delta z^{k}}{k !} \quad \text { and } \\
F(u, v, h) & =\sum_{i=0}^{\infty} \epsilon^{i} F_{i}(u, v, h)
\end{aligned}
$$

in (4). Express the right-hand side of (4) as a polynomial in $\epsilon$. Then, (4) becomes

$$
\begin{aligned}
f_{0}(x, y, \Delta z)= & \frac{1}{4 \pi^{2}} \int_{-}^{+} \int_{\pi}^{\pi} A(u, v, \eta(x, y)) \\
& \cdot e^{s h} e^{j(u x+v y)} d u d v
\end{aligned}
$$

where

$$
\begin{aligned}
A[u, v, & \eta(x, y)] \\
= & F_{0}(u, v, h)-\epsilon\left[\eta(x, y) s F_{0}(u, v, h)-F_{1}(u, v, h)\right] \\
& +\epsilon^{2}\left[\frac{\eta^{2}(x, y)}{2 !} s^{2} F_{0}(u, v, h)-\eta(x, y)\right. \\
& \left.\cdot s F_{1}(u, v, h)+F_{2}(u, v, h)\right] \\
& -\epsilon^{3}\left[\frac{\eta^{3}(x, y)}{3 !} s^{3} F_{0}(u, v, h)-\frac{\eta^{2}(x, y)}{2 !}\right. \\
& \cdot s^{2} F_{1}(u, v, h)+\frac{\eta(x, y)}{1} \\
& \left.\cdot s F_{2}(u, v, h)-F_{3}(u, v, h)\right]+\cdots
\end{aligned}
$$

We set the coefficients of $\epsilon$ to zero giving us the following set of interconnected equations from which we can obtain $F_{k}(u, v, h), k=0,1,2, \cdots$. For example,

$$
\begin{aligned}
F_{0}(u, v, h)= & e^{-s h} \sum_{x} \sum_{y} f_{0}(x, y, \Delta z) e^{-j(u x+v y)} \\
F_{1}(u, v, h)= & e^{-s h} \sum_{x} \sum_{y} \eta(x, y) e^{-j(u x+v y)} \frac{1}{4 \pi^{2}} \\
& \cdot \int_{-}^{+} \int_{\pi}^{\pi} F_{0}(u, v, h) s e^{s h} e^{j(u x+v y)} d u d v \\
F_{2}(u, v, h)= & e^{-s h}\left[\sum_{x} \sum_{y} \eta(x, y) e^{-j(u x+v y)} \frac{1}{4 \pi^{2}}\right. \\
& \cdot \int_{-}^{+} \int_{\pi}^{\pi} F_{1}(u, v, h) s e^{s h} e^{j(u x+v y)} d u d v \\
& -\sum_{x} \sum_{y} \eta^{2}(x, y) e^{-j(u x+v y)} \frac{1}{4 \pi^{2}} \\
& \left.\cdot \int_{-}^{+} \int_{\pi}^{\pi} F_{0}(u, v, h) \frac{s^{2}}{2 !} e^{s h} e^{j(u x+v y)} d u d v\right]
\end{aligned}
$$

and so on. The above system of iterating equations is easily evaluated using the FFT algorithm. The convergence is rapid, usually requiring about 10 iterations, depending upon the amplitude of the surface undulations.

\section{Computer Simulation}

For the purposes of testing the new method of reduction to a horizontal surface we have a synthetically generated field along a set of 16 nearly parallel lines placed on the 


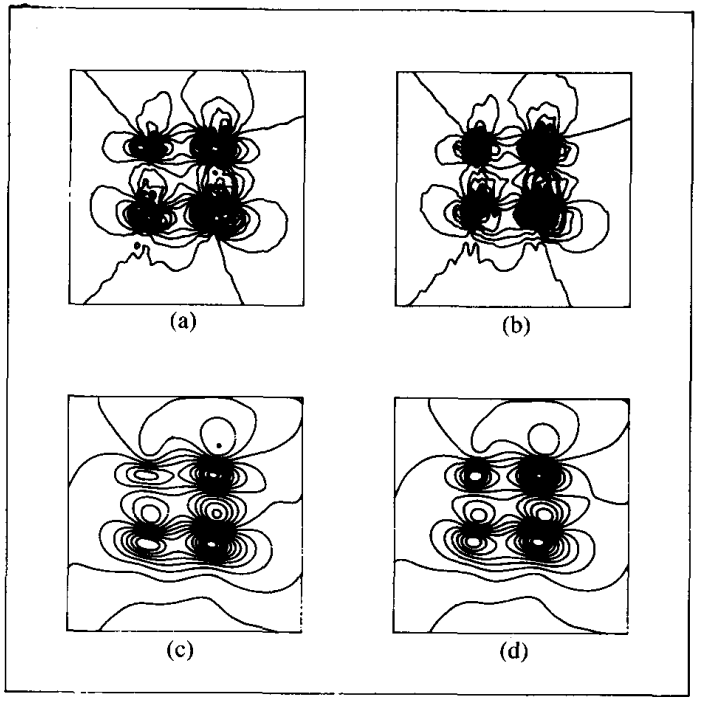

Fig. 3. The contoured maps of measured field are shown in (a) (parallel flight lines) and (b) (scattered stations). The results of field reduction to a horizontal plane at 7.5 units above using the new method are shown in (c) and (d). Compare the reduced field with the theoretical field shown in Fig. 4.

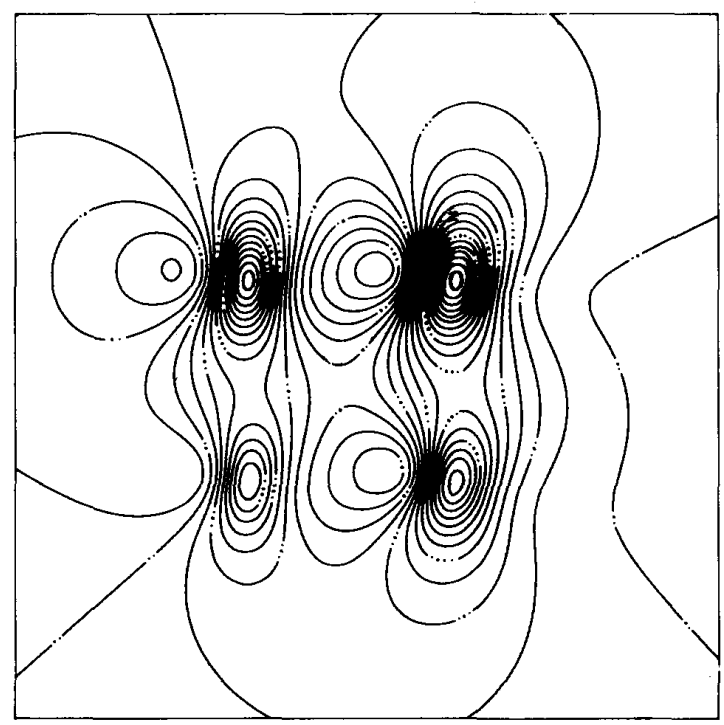

Fig. 4. The theoretically computed field on the plane of reduction.

undulating surface, as well as at randomly scattered (2500) points (see Fig. 2). The parallel lines would correspond to undulating flight paths of an aircraft over a hilly terrain. On each line 160 samples were collected, giving a total of 2560 samples. The data thus created was gridded using a commercially available software package (e.g., Data Plotting Services Inc., Toronto, Ont., Canada). The contoured maps are shown in Fig. 3(a) and (b). The result of field reduction to a horizontal plane at 7.5 units above using the new method is shown in Fig. 3(c) and (d). The theoretically computed field on the plane of reduction is shown in Fig. 4. Table II gives the rms difference be-

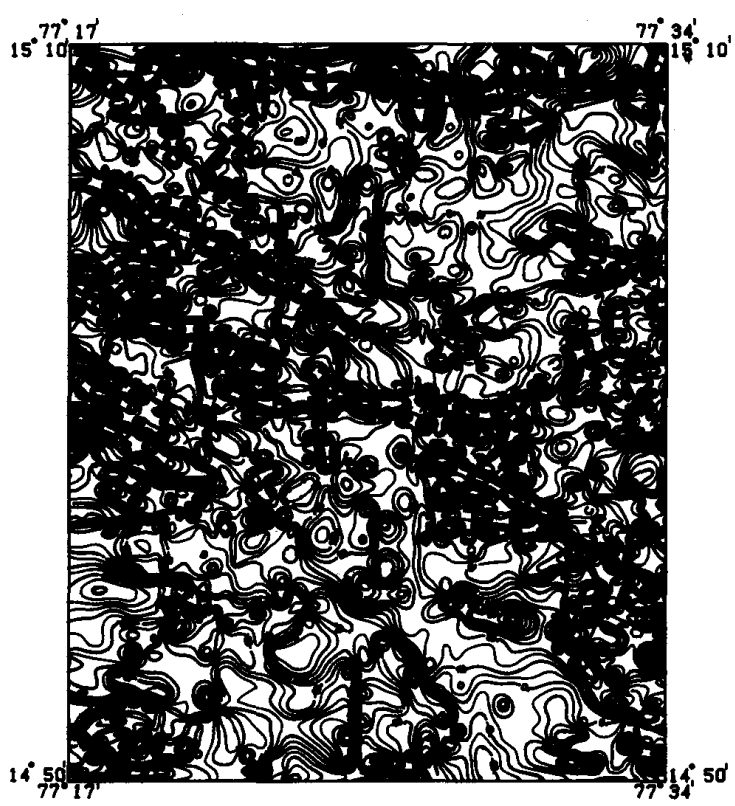

Fig. 5. Aeromagnetic field over a hilly terrain flown at low altitude (nominal height $50 \mathrm{~m}$ above ground). Contour interval is $20 \gamma$.

Table II

\begin{tabular}{|l|c|c|}
\hline $\begin{array}{c}\text { Nature of } \\
\text { Data } \\
\text { (2500 points) }\end{array}$ & $\begin{array}{c}\text { Cpu time in } \\
\text { minutes on } \\
\operatorname{vax} 750\end{array}$ & $\begin{array}{c}\text { RMS error } \\
\text { in \% }\end{array}$ \\
\hline $\begin{array}{l}\text { Scattered } \\
\text { Data }\end{array}$ & 12.0 & 2.59 \\
\hline $\begin{array}{l}\text { Parallel } \\
\text { Profiles }\end{array}$ & 12.0 & 6.36 \\
\hline Grid data & 8.0 & 1.44 \\
\hline
\end{tabular}

tween the reduced and the theoretical field. We note that in comparison with the least-squares methods there is a marginal increase in the rms error; however, there is a considerable savings in computer time, by a factor of 10 to 12 . The increase in the rms error is largely due to the interpolation error. In support of this statement we have carried out the reduction to a horizontal plane using data on a regular grid requiring no gridding. From the last row in Table II we note that the rms error has come down and is of the same magnitude as in the least-squares approach.

\section{Application to Real Data}

We have applied our new method of reduction to a real data set obtained in a low altitude (nominal flight height $50 \mathrm{~m}$ ) aeromagnetic survey carried over a part of the diamond-bearing rocks, consisting of granites and granite gneisses belonging to the south Indian shield. The survey was carried out by the AMSE division of the Geological Survey of India using the Scintrex system mounted on a Twin Otter aircraft. In addition to the total magnetic field, the system provided continuous measurements of barometric height and air column thickness, which were used 


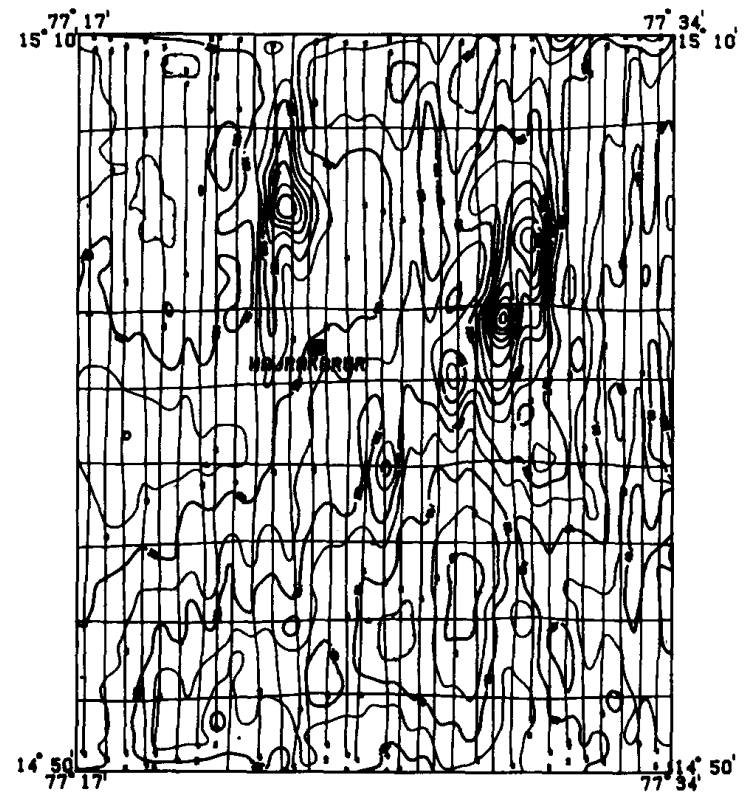

Fig. 6. Reconstructed flight surface from the barometric data along with the flight paths. Contour interval is $20 \mathrm{~m}$.

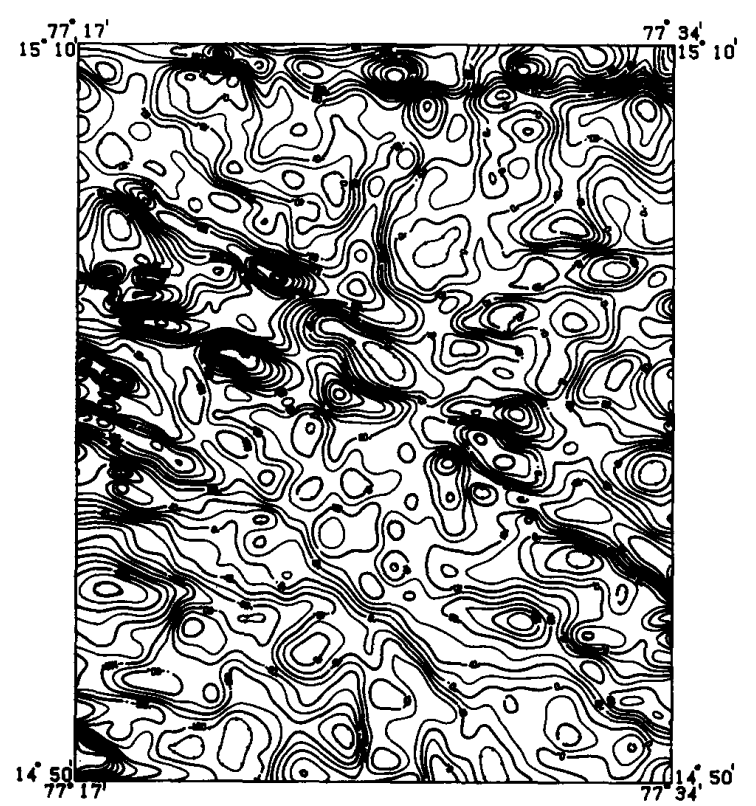

Fig. 7. Total field reduced to a horizontal plane at a height of $678 \mathrm{~m}$ above MSL. The plane of reduction is just above the highest point in the survey area.

to reconstruct the actual flight surface. The navigation system consisted of Doppler as well as a visual method based on aerial photostrips. It is believed that the positional accuracy is better than $5 \mathrm{~m}$. The total measured field after the application of the diurnal and instrumental drift corrections and subtracting a base level of $41100 \gamma$ is shown in Fig. 5 and the reconstructed flight surface is shown in Fig. 6. On the flight surface we have also superposed actual flight lines. The contour values are in me- ters, representing the height above MSL plus $297 \mathrm{~m}$. In Fig. 7 we show the reduced field, which now corresponds to what would have been observed on a horizontal plane at a height of $678 \mathrm{~m}$ above MSL or $381 \mathrm{~m}$ above the local base level.

\section{ACKNOWLEDGMENT}

We wish to thank the Director-General of the Geological Survey of India, Calcutta, for permission to use their aeromagnetic data. We also wish to thank M. R. Nair, Deputy DG, AMSE, for his interest in this work and $M$. V. Ramanmurthy, R. Sharma, Dr. S. V. Anand, P. V. Anil Kumar, M. Singh, and B. Singh, all from AMSE, for their help in preparing the maps.

\section{REFERENCES}

[1] V. N. Strakhov and V. M. Devitsyn, "The reduction of observed values of a potential field to values at a constant level," Bull. (Izv.) Acad. Sci. U.S.S.R., Earth Physics, no. 4, pp. 256-261, 1965.

[2] A. V. Tsirul'skiy, "On the reduction of observed values of potential fields to a single level," Bull. (Izv.) Acad. Sci. U.S.S.R., Earth Physics, no. 3, 1968.

[3] A. V. Tsirul'skiy and L. Ya. Ospishcheva, "An algorithm for the reduction of observed potential field values to a common level," Bull. (Izv.) Acad. Sci. U.S.S.R., Earth Physics, no. 4, pp. 266-270, 1968.

[4] B. K. Bhattacharya and K. C. Chan, "Reduction of magnetic and gravity data on an arbitrary surface acquired in a region of high topographic relief,"' Geophys., vol. 42, pp. 1411-1430, 1977.

[5] J. C. Wynn and B. K. Bhattacharya, "Reduction of terrain induced aeromagnetic anomalies by parallel surface continuation: A case history from southern San Juan mountains of Colorado," Geophys., vol. 42, pp. 1431-1449, 1977.

[6] R. O. Hansen and Y. Miyazaki, "Continuation of potential fields between arbitrary surfaces," Geophys., vol. 49, pp. 787-795, 1984

[7] R. G. Henderson and L. Cordell, "Reduction of unevenly spaced potential field data to a horizontal plane by means of finite harmonic series," Geophys., vol. 36, pp. 856-866, 1971.

[8] V. Courtillot, J. Ducruix, and J. L. Le Mouel, "Le prolongement d'un champ de potentiel d'un contour quelconque sur un contour horizontal: Une application del la method de Backus et Gilbert," Ann. Geophys., vol. 29, pp. 361-366, 1973.

[9] J. Ducruix, J L. Le Mouel, and V. Courtillot, "Continuation of three dimensional potential fields measured on an uneven surface," Geophys. J. R. Astr. Soc., vol. 38, pp. 299-314, 1974

[10] R. L. Parker and K. D. Klitgord, "Magnetic upward continuation from an uneven track," Geophys., vol. 37, pp. 662-668, 1972.

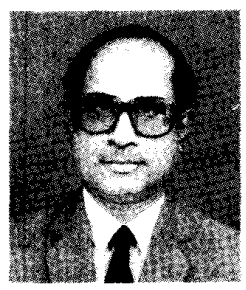

P. S. Naidu was born in 1937 . He received the M.S. degree from the Indian Institute of Technology, Kharagpur, and the Ph.D. degree from the University of British Columbia, Vancouver, Canada

Currently, he is a Professor of Electrical Communication Engineering at the Indian Institute of Science, Bangalore. He has published over seventy research papers in geophysical and underwater signal processing.

Dr. Naidu was a recipient of a Humboldt Fellowship (1979) and an NRC Senior Associate (1988).

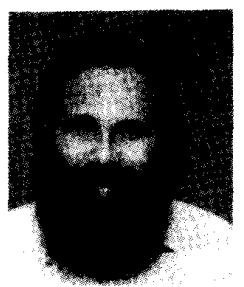

M. P. Mathew was born in 1947 . He received the M.S. degree in mathematics and statistics from the Kerala University.

He joined the Geological Survey of India in 1970 and performed gravity and magnetic surveys. Currently, he is with the Airborne Mineral Survey and Exploration wing of the GSI where he is engaged in aeromagnetic data processing. 